\title{
Association between Resting Heart Rate and Inflammatory Markers (White Blood Cell Count and High-Sensitivity C-Reactive Protein) in Healthy Korean People
}

\author{
Woo-Chul Park', Inho Seo', Shin-Hye Kim', Yong-Jae Lee ${ }^{1, *}$, Song Vogue Ahn ${ }^{2, *}$ \\ ${ }^{1}$ Department of Family Medicine, Yonsei University College of Medicine, Seoul, Korea \\ ${ }^{2}$ Department of Preventive Medicine, Institute of Genomic Cohort, Yonsei University Wonju College of Medicine, Wonju, Korea
}

\begin{abstract}
Background: Inflammation is an important underlying mechanism in the pathogenesis of atherosclerosis, and an elevated resting heart rate underlies the process of atherosclerotic plaque formation. We hypothesized an association between resting heart rate and subclinical inflammation.

Methods: Resting heart rate was recorded at baseline in the KoGES-ARIRANG (Korean Genome and Epidemiology Study on Atherosclerosis Risk of Rural Areas in the Korean General Population) cohort study, and was then divided into quartiles. Subclinical inflammation was measured by white blood cell count and high-sensitivity C-reactive protein. We used progressively adjusted regression models with terms for muscle mass, body fat proportion, and adiponectin in the fully adjusted models. We examined inflammatory markers as both continuous and categorical variables, using the clinical cut point of the highest quartile of white blood cell count $\left(\geq 7,900 / \mathrm{mm}^{3}\right)$ and $\geq 3 \mathrm{mg} / \mathrm{dL}$ for high-sensitivity C-reactive protein.

Results: Participants had a mean age of $56.3 \pm 8.1$ years and a mean resting heart rate of $71.4 \pm 10.7$ beats $/ \mathrm{min} ; 39.1 \%$ were men. In a fully adjusted model, an increased resting heart rate was significantly associated with a higher white blood cell count and higher levels of high-sensitivity C-reactive protein in both continuous ( $\mathrm{P}$ for trend $<0.001$ ) and categorical (P for trend $<0.001$ ) models.

Conclusion: An increased resting heart rate is associated with a higher level of subclinical inflammation among healthy Korean people.
\end{abstract}

Keywords: Heart Rate; Leukocytes; C-Reactive Protein; Inflammation; Atherosclerosis

\footnotetext{
Received: February 24, 2016, Revised: May 2, 2016, Accepted: May 19, 2016

*Corresponding Author: Yong-Jae Lee Tel: +82-2-2019-3480, Fax: +82-2-3463-3287, E-mail: ukyjhome@yuhs.ac

*Corresponding Author: Song Vogue Ahn Tel: +82-33-741-0348, Fax: +82-33-747-0409, E-mail: dodge@yonsei.ac.kr

*These authors contributed equally to this work are co-corresponding authors.
} 


\section{INTRODUCTION}

Inflammation is an important underlying mechanism in the pathogenesis of atherosclerosis. ${ }^{1,2)}$ Subclinical inflammation and levels of various inflammatory markers correlate strongly with cardiovascular morbidity and mortality in both healthy individuals and those with known coronary artery disease (CAD)., ${ }^{3,4)}$ The levels of various inflammatory markers, including white blood cell (WBC) count and levels of high-sensitivity C-reactive protein (hs-CRP), have proved to be independently associated with atherosclerosis, as represented by the angiographic confirmation of $\mathrm{CAD}$. $^{5-7)}$

Epidemiological evidence indicates that an elevated resting heart rate (HR) is an independent marker of both all-cause and cardiovascular-specific mortality. ${ }^{8-12)}$ An elevated resting HR is also associated with endothelial dysfunction, ${ }^{13)}$ severity of $\mathrm{CAD},{ }^{14,15)}$ and eventually plaque rupture, ${ }^{16)}$ indicating that an elevated HR underlies every process of atherosclerotic plaque formation.

Previous epidemiologic studies have demonstrated that HR is associated with inflammatory biomarkers, but those studies were limited either by missing covariates, such as body composition and adiponectin, or by the inclusion of participants with cardiovascular disease. ${ }^{17-20)}$ Thus, we hypothesized that an elevated resting HR is associated with inflammation, as measured by 2 different markers (WBC count and hs-CRP), and we evaluated this hypothesis by using multivariate adjusted analysis in healthy Korean people without apparent cardiovascular disease.

\section{METHODS}

\section{Study Population}

We used data from the baseline visit (2005-2007) of the Korean Genome and Epidemiology Study on Atherosclerosis Risk of Rural Areas in the Korean General Population (KoGES-ARIRANG), a populationbased prospective cohort study designed to assess the prevalence, incidence, and risk factors for chronic degenerative disorders, such as hypertension, diabetes, osteoporosis, and cardiovascular disease. The baseline examinations were carried out in the rural areas of Wonju and Pyeongchang from November 2005 to January 2008.

Of the 10,107 adults who comprised the KoGES-ARIRANG cohort, we excluded 2506 individuals because of a missing resting HR value, WBC count, or hs-CRP value ( $\mathrm{n}=351)$; a history of cerebrovascular disease $(n=204)$ or heart disease $(n=313)$; or other missing data $(n=706)$. To eliminate individuals with acute phase inflammation, we excluded individuals with a clinically abnormal WBC count (more than 10,000/ $\mathrm{mm}^{3} ; \mathrm{n}=757$ ) or an hs-CRP level $\geq 10 \mathrm{mg} / \mathrm{L}$ ( $\mathrm{n}=175$ ). Finally, 7,601 participants (2,975 men, 4,626 women) were analyzed.

\section{Data Collection}

At baseline and follow-up examinations, study subjects completed a standardized medical history and lifestyle questionnaire and underwent a comprehensive health examination according to standard pro- cedures. Body weight and height were measured while subjects wore light indoor clothing without shoes. Waist circumference was measured in a horizontal plane, midway between the inferior margin of the ribs and the superior border of the iliac crest, using a tape measure (SECA-200; SECA, Hamburg, Germany). Muscle mass and body fat proportion were measured by bioelectrical impedance analysis (Zeus 9.9; Jawon Medical Co. Ltd., Kyoungsan, Korea). Participants' resting HR was recorded during the baseline examination, using an automatic blood pressure monitor (FT-500, Jawon Medical Co. Ltd.). The automatic blood pressure monitor measured the frequency of the heartbeat and time duration while measuring the blood pressure, and the heartbeats per minute were calculated. Systolic blood pressure (SBP) and diastolic blood pressure (DBP) were measured twice in the right arm by using a standard mercury sphygmomanometer (Baumanometer, Copiague, NY, USA). The mean of the 2 blood pressure readings was used for data analyses. Smoking status was determined based on self-report. Nonsmokers were defined as subjects who had smoked $<100$ cigarettes ( $<5$ packs of cigarettes) in their lifetime. Current smokers were defined as subjects who had smoked $\geq 100$ cigarettes in their lifetime and who reported that they were 'currently smoking' in the questionnaire. Former smokers were defined as subjects who had smoked $\geq 100$ cigarettes in their lifetime but who reported that they 'abstain from smoking' in the questionnaire.

A venous blood sample was drawn from study participants after a 12-hour or overnight fast. Serum concentrations of adiponectin were measured by radioimmunoassay (LINCO Research Inc., St. Charles, MO, USA), with intra-assay coefficients of variation ranging between $2.9 \%$ and $6.6 \%$. Fasting glucose was determined by a glucose-oxidase based assay. Serum concentrations of low-density lipoprotein (LDL) cholesterol, high-density lipoprotein (HDL) cholesterol, and triglycerides were determined by enzymatic methods (Avida 1650, Siemens, Tarrytown, NY, USA). WBC count was measured using an automated blood cell counter (ADVIA 120, Bayer, NY, USA). hs-CRP was measured by the Denka Seiken (Tokyo, Japan) assay, which has been validated against the Dade Behring method.

Hypertension was defined as SBP $\geq 140 \mathrm{~mm} \mathrm{Hg}$ or DBP $\geq 90 \mathrm{~mm} \mathrm{Hg}$, or a self-report of hypertension, or the use of an antihypertensive medication; diabetes mellitus was defined as a fasting plasma glucose level $\geq 126 \mathrm{mg} / \mathrm{dL}$, or glycated hemoglobin (HbAlc) $\geq 6.5 \%$, or clinically known and treated diabetes mellitus.

\section{Statistical Analyses}

Participants were divided into resting HR quartiles (Q1: $\mathrm{n}=2,039, \mathrm{Q} 2$ : $\mathrm{n}=1,817, \mathrm{Q} 3: \mathrm{n}=2,020, \mathrm{Q} 4: \mathrm{n}=1,725)$. Baseline characteristics for the continuous variables were expressed as means \pm standard deviations, and comparisons between groups were performed using a 1-factor analysis of variance. Categorical data are summarized as percentages, and comparisons between groups were performed using the Pearson chi-square test.

Inflammation was examined as a continuous variable using linear regression models. hs-CRP was not normally distributed and was 
therefore log transformed for continuous analyses. In categorical analyses of the inflammatory variables, we used the clinical cut point for a normal hs-CRP $<3 \mathrm{mg} / \mathrm{dL}$ as the reference group and an elevated hs$\mathrm{CRP} \geq 3 \mathrm{mg} / \mathrm{dL}$ as the subclinical inflammation group. To set the cut point of WBC count, the study population was divided into WBC count quartiles. We set the highest quartile of $\mathrm{WBC}$ count $\left(\geq 7,900 / \mathrm{mm}^{3}\right)$ as the subclinical inflammation group, with the three lowest WBC count quartiles as the reference group.

For each of our secondary analyses, we used these categorical cut points to calculate the odds ratio (OR) and $95 \%$ confidence interval
(CI). The results of these analyses are interpreted as the OR of having an elevated inflammatory level compared with a non-elevated level for each HR quartile, with the lowest HR quartile as the reference. To evaluate the adjusted $\mathrm{OR}$, we ran 3 multivariate logistic regression models. Model I included age and sex. Model 2 added smoking status, SBP, DBP, fasting plasma glucose, HbAlc, LDL and HDL cholesterol, triglycerides, body mass index, waist circumference, hypertension, diabetes mellitus, and lipid-lowering medication use. Model 3 added muscle mass, body fat proportion, and serum adiponectin level.

The level of significance used for all of the above analyses was a

Table 1. Participant characteristics by baseline resting heart rate quartile

\begin{tabular}{|c|c|c|c|c|c|}
\hline \multirow{2}{*}{ Characteristic } & \multicolumn{4}{|c|}{ Baseline resting heart rate quartile } & \multirow{2}{*}{ P for treno } \\
\hline & 1 & 2 & 3 & 4 & \\
\hline Resting heart rate (beats/min) & $59.4(36,64)$ & $67.6(65,70)$ & $74.3(71,78)$ & $86.4(79,129)$ & $<0.001$ \\
\hline Age (y) & $56.9 \pm 7.8$ & $56.2 \pm 8.1$ & $55.5 \pm 8.2$ & $56.5 \pm 8.4$ & 0.016 \\
\hline Men (\%) & 48.2 & 36.0 & 35.2 & 36.3 & $<0.001$ \\
\hline Current smoker (\%) & 14.6 & 13.8 & 14.7 & 17.0 & 0.039 \\
\hline Systolic blood pressure (mm Hg) & $129.8 \pm 18$ & $130.1 \pm 18$ & $130.1 \pm 18$ & $132.6 \pm 19$ & $<0.001$ \\
\hline Diastolic blood pressure $(\mathrm{mm} \mathrm{Hg})$ & $81.1 \pm 11$ & $82.1 \pm 11$ & $82.5 \pm 11$ & $85.0 \pm 12$ & $<0.001$ \\
\hline Fasting plasma glucose (mg/dL) & $94.0 \pm 20$ & $94.6 \pm 19$ & $96.6 \pm 23$ & $101.5 \pm 33$ & $<0.001$ \\
\hline Glycated hemoglobin (\%) & $5.6 \pm 0.8$ & $5.6 \pm 0.8$ & $5.6 \pm 0.8$ & $5.8 \pm 1.0$ & $<0.001$ \\
\hline Low-density lipoprotein (mg/dL) & $118.0 \pm 32$ & $119.6 \pm 32$ & $120.1 \pm 33$ & $119.6 \pm 35$ & 0.119 \\
\hline High-density lipoprotein (mg/dL) & $46.2 \pm 11$ & $46.4 \pm 11$ & $46.3 \pm 11$ & $46.2 \pm 11$ & 0.889 \\
\hline Triglycerides (mg/dL) & $120(82-177)$ & $124(87-177)$ & $126(88-187)$ & $140(96-205)$ & $<0.001$ \\
\hline Body mass index $\left(\mathrm{kg} / \mathrm{m}^{2}\right)$ & $24.4 \pm 3.0$ & $24.4 \pm 3.0$ & $24.4 \pm 3.2$ & $24.3 \pm 3.4$ & 0.806 \\
\hline Waist circumference (cm) & $83.4 \pm 8.2$ & $83.0 \pm 8.5$ & $82.9 \pm 8.7$ & $83.8 \pm 8.7$ & 0.275 \\
\hline Hypertension (\%) & 39.8 & 39.8 & 40.4 & 45.4 & 0.001 \\
\hline Diabetes mellitus (\%) & 11.9 & 11.5 & 12.7 & 17.1 & $<0.001$ \\
\hline Lipid-lowering medication (\%) & 2.1 & 1.9 & 1.7 & 1.0 & 0.017 \\
\hline Muscle mass $(\mathrm{kg})$ & $41.2 \pm 7.5$ & $40.0 \pm 7.1$ & $39.8 \pm 7.2$ & $39.5 \pm 7.3$ & $<0.001$ \\
\hline Body fat proportion (\%) & $28.0 \pm 6.8$ & $29.0 \pm 6.2$ & $29.2 \pm 6.5$ & $29.0 \pm 6.9$ & $<0.001$ \\
\hline Adiponectin $(\mu \mathrm{g} / \mathrm{mL})$ & $9.60(6.58-13.33)$ & $9.68(6.50-13.35)$ & $9.46(6.25-13.16)$ & $9.22(6.35-12.94)$ & 0.026 \\
\hline White blood cell (cells/mm³) & $6,540 \pm 1,500$ & $6,600 \pm 1,540$ & $6,700 \pm 1,500$ & $6,970 \pm 1,520$ & $<0.001$ \\
\hline High-sensitivity C-reactive protein (mg/dL) & $0.73(0.39-1.47)$ & $0.72(0.37-1.44)$ & $0.78(0.41-1.63)$ & $0.84(0.44-1.80)$ & $<0.001$ \\
\hline
\end{tabular}

Values are presented as mean (minimum, maximum), mean \pm standard deviation, \%, or median (interquartile range), unless otherwise indicated.

A

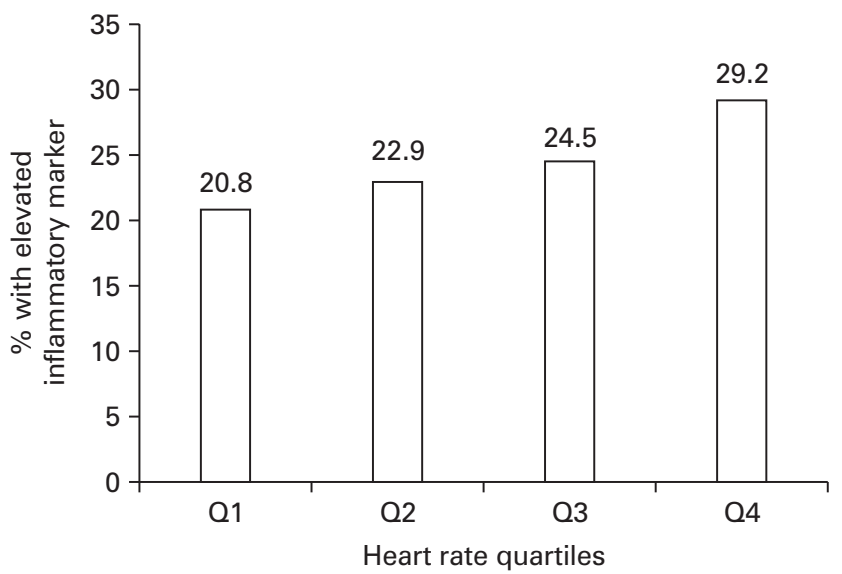

B

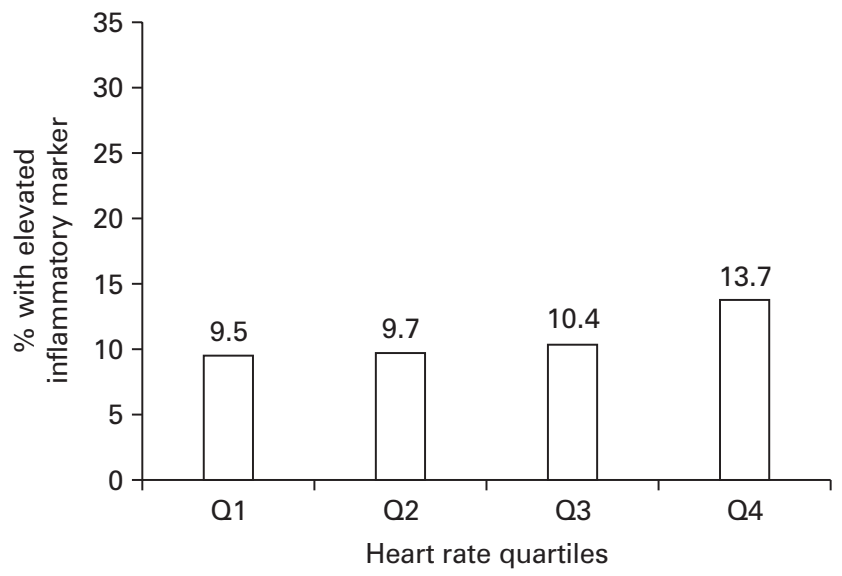

Figure 1. Percentage of participants in the upper quartile (A) of white blood cell $\left(\geq 7,900 / \mathrm{mm}^{3}\right)$ or $(B)$ with an high sensitivity C-reactive protein $\geq 3.0$ mg/dL by resting heart rate quartile. 
2-tailed P-value $<0.05$. All calculations were performed using PASW SPSS ver. 18.0 (SPSS Inc., Chicago, IL, USA).

\section{RESULTS}

At baseline examination, participants with a higher resting HR were more likely to be women, and more likely to have hypertension or diabetes mellitus, higher blood pressure, higher levels of fasting plasma glucose, $\mathrm{HbAlc}$, and triglycerides, and a greater body fat proportion (Table 1). Mean values of WBC count and hs-CRP level were higher among those with higher resting HR. Participants with a lower resting HR were more likely to be men, to have more muscle mass, to be taking a lipid-lowering medication, and to have a higher adiponectin level.

For both inflammatory markers, an increase in resting HR was asso-

Table 2. Estimated increase in WBC counts and levels of hs-CRP, stratified by baseline resting heart rate quartile

\begin{tabular}{|c|c|c|c|c|c|}
\hline \multirow{2}{*}{ Marker } & \multicolumn{4}{|c|}{ Resting heart rate quartile, $\beta$ coefficient } & \multirow{2}{*}{ P for trend } \\
\hline & 1 & 2 & 3 & 4 & \\
\hline \multicolumn{6}{|c|}{ WBC (cells $/ \mathrm{mm}^{3}$ ) } \\
\hline Unadjusted & Reference & 0.017 & $0.048^{*}$ & $0.117^{*}$ & $<0.001$ \\
\hline Model 1 & Reference & $0.037^{*}$ & $0.070^{\star}$ & $0.135^{\star}$ & $<0.001$ \\
\hline Model 2 & Reference & $0.030^{*}$ & $0.056^{*}$ & $0.109^{*}$ & $<0.001$ \\
\hline Model 3 & Reference & $0.030^{*}$ & $0.053^{*}$ & $0.105^{\star}$ & $<0.001$ \\
\hline \multicolumn{6}{|l|}{$\mathrm{hs}-\mathrm{CRP}(\mathrm{mg} / \mathrm{L})^{\dagger}$} \\
\hline Unadjusted & Reference & -0.009 & 0.022 & $0.061^{*}$ & $<0.001$ \\
\hline Model 1 & Reference & 0.009 & $0.048^{*}$ & $0.075^{\star}$ & $<0.001$ \\
\hline Model 2 & Reference & 0.004 & $0.037^{\star}$ & $0.053^{*}$ & $<0.001$ \\
\hline Model 3 & Reference & 0.005 & $0.035^{*}$ & $0.051^{*}$ & $<0.001$ \\
\hline
\end{tabular}

Model 1: age and sex; model 2: model 1+smoking, systolic blood pressure, diastolic blood pressure, fasting plasma glucose, glycated hemoglobin, low-density lipoprotein cholesterol, high-density lipoprotein cholesterol, triglycerides, body mass index, waist circumference, hypertension, diabetes mellitus, and lipid-lowering medication; model 3: model 2+muscle mass, body fat proportion, and serum adiponectin level. WBC, white blood cell; hs-CRP, high-sensitivity C-reactive protein.

${ }^{*} \mathrm{P}<0.05$. 'Presented using log-transformed values. ciated with an increased percentage of subjects who had an elevated inflammatory level. The absolute percentage of subjects classified with an elevated inflammatory level was higher for WBC count $(29.2 \%$ in quartile 4) (Figure 1A) than for hs-CRP (13.7\% in quartile 4) (Figure 1B).

In linear regression models, WBC count increased with increasing HR after multivariate adjustment ( $P$ for trend $<0.001$ ) (Table 2 ), and it is notable that the increment in the $\beta$ coefficient was much larger in the fourth quartile than in other quartiles. Although the difference in $\beta$ coefficient between the second and reference quartiles was not statistically significant, hs-CRP also showed a tendency to increase with increasing HR after multivariate adjustment (P for trend $<0.001$ ) (Table 2).

Participants with a higher resting HR also had a progressively increased risk of having an elevated inflammatory marker compared with those with a lower resting HR ( $P$ for trend $<0.001$ for both markers) (Table 3). In the fully adjusted model, the OR for being in the upper quartile of WBC count $\left(\geq 7,900 / \mathrm{mm}^{3}\right)$ was significantly increased for all resting HR quartiles. The OR for having an hs-CRP level $\geq 3 \mathrm{mg} /$ $\mathrm{dL}$ was also increased for all resting HR quartiles, but it was only statistically significant for the fourth quartile compared to the first. For the highest quartile of resting $\mathrm{HR}$, the OR for having an elevated inflammatory marker was similar across the 2 inflammatory markers (OR, 1.527; $95 \%$ CI, 1.303 to 1.789 for WBC; OR, 1.555 ; 95\% CI, 1.258 to 1.923 for hs-CRP). The association between resting HR and both inflammatory markers showed amplification, which means that the increment in the OR was much larger in the fourth quartile compared with other quartiles.

\section{DISCUSSION}

A number of epidemiologic studies have demonstrated that resting HR is associated with inflammatory biomarkers. Rogowski et al. ${ }^{17)}$ reported that resting HR was significantly associated with a micro-inflammatory response (fibrinogen, hs-CRP, and polymorphonuclear leukocyte

Table 3. ORs and $95 \%$ Cls for being in the upper quartile of WBC counts $\left(\geq 7,900 / \mathrm{mm}^{3}\right)$ or having a hs-CRP level $\geq 3 \mathrm{mg} / \mathrm{dL}$

\begin{tabular}{|c|c|c|c|c|c|}
\hline \multirow{2}{*}{ Marker } & \multicolumn{4}{|c|}{ Resting heart rate quartile } & \multirow{2}{*}{ P for trend } \\
\hline & 1 & 2 & 3 & 4 & \\
\hline \multicolumn{6}{|l|}{ WBC (cells $/ \mathrm{mm}^{3}$ ) } \\
\hline Unadjusted & Reference & 1.135 (0.974-1.322) & $1.233(1.064-1.429)^{*}$ & $1.568(1.351-1.820)^{*}$ & $<0.001$ \\
\hline Model 1 & Reference & $1.223(1.047-1.427)^{\star}$ & $1.342(1.155-1.558)^{*}$ & $1.691(1.454-1.967)^{*}$ & $<0.001$ \\
\hline Model 2 & Reference & $1.196(1.022-1.400)^{*}$ & $1.283(1.101-1.495)^{*}$ & $1.561(1.333-1.827)^{*}$ & $<0.001$ \\
\hline Model 3 & Reference & $1.198(1.023-1.403)^{*}$ & $1.270(1.089-1.480)^{*}$ & $1.527(1.303-1.789)^{*}$ & $<0.001$ \\
\hline \multicolumn{6}{|l|}{ hs-CRP (mg/L) } \\
\hline Unadjusted & Reference & $1.026(0.828-1.272)$ & $1.116(0.908-1.370)$ & $1.523(1.245-1.864)^{*}$ & $<0.001$ \\
\hline Model 1 & Reference & $1.084(0.873-1.346)$ & 1.205 (0.979-1.483) & $1.596(1.302-1.957)^{\star}$ & $<0.001$ \\
\hline Model 2 & Reference & $1.082(0.869-1.346)$ & $1.198(0.970-1.478)$ & $1.564(1.266-1.933)^{\star}$ & $<0.001$ \\
\hline Model 3 & Reference & $1.087(0.873-1.354)$ & $1.192(0.965-1.473)$ & $1.555(1.258-1.923)^{\star}$ & $<0.001$ \\
\hline
\end{tabular}

Values are presented as OR $(95 \% \mathrm{Cl})$. Model 1: age and sex; model 2: model 1+smoking status, systolic blood pressure, diastolic blood pressure, fasting plasma glucose, HbA1c, low-density lipoprotein cholesterol, high-density lipoprotein cholesterol, triglycerides, body mass index, waist circumference, hypertension, diabetes mellitus, and lipidlowering medication; model 3: model 2+muscle mass, body fat proportion, and serum adiponectin level.

$\mathrm{OR}$, odds ratio; $\mathrm{Cl}$, confidence interval; $\mathrm{WBC}$, white blood cell; hs-CRP, high-sensitivity C-reactive protein.

${ }^{*} \mathrm{P}<0.05$. 
count) in a cohort of 4,553 healthy men and in those with atherothrombotic risk factors. In the Okinawa General Health Maintenance Association cohort of 8,772 participants, there was an independent relationship between elevated resting $\mathrm{HR}$ and WBC count in middleaged and elderly healthy Japanese individuals. ${ }^{18)}$ Ó Hartaigh et al. ${ }^{19)}$ found that elevated inflammatory biomarkers (interleukin-6, hs-CRP, serum amyloid A, neutrophils, and fibrinogen) were associated with cardiovascular mortality and that the effects of inflammation appeared to be strongly amplified by an increased resting HR. Moreover, Whelton et al. ${ }^{20)}$ reported that an increased resting HR was associated with higher levels of inflammatory biomarkers (hs-CRP, interleukin-6, and fibrinogen) among an ethnically diverse group of subjects without known cardiovascular disease.

The present study assessed the interrelationship between resting HR and subclinical inflammation. Our findings indicated that resting HR was significantly associated with increased levels of inflammation, as measured by 2 different inflammatory biomarkers. Consistently with previous studies, we found that an increased resting HR was associated with adverse cardiovascular risk factors, including an increase in blood pressure, triglycerides, diabetes, and body fat proportion. However, our results show that the association of HR with inflammation was independent of these risk factors.

Compared with individuals in the first quartile of resting HR, which was the reference group of this study, the OR for the fourth quartile was approximately 1.5 for both WBC count and hs-CRP. Thus, individuals with a resting HR of more than 79 beats per minute had a higher risk of subclinical inflammation, and hence of the subsequent development of atherosclerosis and future cardiovascular events. Our results are compatible with previous epidemiological evidence that a resting HR of more than 75 beats per minute is hazardous for cardiovascular mortality. ${ }^{19)}$

There are 2 potential mechanisms that link elevated resting HR and subclinical inflammation: sympathetic overactivation and mechanical stress. The autonomic nervous system plays a role in immune system regulation and the inflammatory response. Tumor necrosis factor (TNF) is an inflammatory cytokine that precipitates vascular damage through capillary leakage and microvascular thrombosis. ${ }^{21)}$ Vagal nerve stimulation attenuates inflammation by downregulating the release of TNF and other cytokines from macrophages. ${ }^{22,23)}$

Another mechanism that links elevated resting HR with subclinical inflammation might be mechanical stress on vascular endothelium. ${ }^{24)}$ There are 2 types of mechanical stress. One is shear stress, a frictional force on the arterial wall, and the other is tensile stress, a radial force that stretches the arterial wall. Elevated HR increases the frequency of oscillatory shear stress to the vascular endothelium, ${ }^{25)}$ which promotes endothelial superoxide anion expression ${ }^{26)}$ and vascular cell adhesion molecule expression, thus enhancing endothelial monocyte adhesion. $^{27-29)}$

One of the strengths of our study is that it is the first large cross-sectional study in Korea to highlight the relationship between resting HR and inflammatory markers in a healthy general population. Moreover, we evaluated our hypothesis by multivariate adjustment, including important covariates such as body composition and adiponectin, which to our knowledge has not been reported by previous analyses.

The present study is not without limitations. Its cross-sectional nature does not provide information with regard to temporality. Although the study was prospective as regards patient enrolment, resting HR was only estimated at a single time point and therefore may not reflect the HR over a longer period. Furthermore, HR shows significant diurnal variation but HR was not always measured at the same time of the day in this study. Similarly, the single measurement of inflammatory markers may not accurately reflect the long-term inflammatory state of an individual participant. Lastly, we did not take into consideration the effect of medicines, such as $\beta$-blockers and non-dihydropyridine calcium channel blockers, on resting HR.

In conclusion, the present study provides evidence that an elevated resting HR was independently associated with increased inflammatory markers in healthy Korean people. Our finding demonstrates the clinical significance of resting HR for identifying individuals at risk of subclinical inflammation and a future cardiovascular event.

\section{CONFLICT OF INTEREST}

No potential conflict of interest relevant to this article was reported.

\section{ACKNOWLEDGMENTS}

This study was supported by a grant of the Korea Centers for Disease Control and Prevention (2005-E71013-00, 2006-E71002-00, and 2007E71013-00).

\section{REFERENCES}

1. Ross R. The pathogenesis of atherosclerosis: a perspective for the 1990s. Nature 1993;362:801-9.

2. Libby P. Inflammation in atherosclerosis. Nature 2002;420:868-74.

3. Morrow DA, Rifai N, Antman EM, Weiner DL, McCabe CH, Cannon $\mathrm{CP}$, et al. C-reactive protein is a potent predictor of mortality independently of and in combination with troponin $\mathrm{T}$ in acute coronary syndromes: a TIMI 11A substudy: thrombolysis in myocardial infarction. J Am Coll Cardiol 1998;31:1460-5.

4. Ridker PM, Cushman M, Stampfer MJ, Tracy RP, Hennekens CH. Inflammation, aspirin, and the risk of cardiovascular disease in apparently healthy men. N Engl J Med 1997;336:973-9.

5. Zebrack JS, Muhlestein JB, Horne BD, Anderson JL; Intermountain Heart Collaboration Study Group. C-reactive protein and angiographic coronary artery disease: independent and additive predictors of risk in subjects with angina. J Am Coll Cardiol 2002;39:632-7.

6. Cavusoglu E, Chopra V, Gupta A, Ruwende C, Yanamadala S, Eng C, et al. Usefulness of the white blood cell count as a predictor of angiographic findings in an unselected population referred for coronary angiography. Am J Cardiol 2006;98:1189-93.

7. Zhang R, Brennan ML, Fu X, Aviles RJ, Pearce GL, Penn MS, et al. As- 
sociation between myeloperoxidase levels and risk of coronary artery disease. JAMA 2001;286:2136-42.

8. Kannel WB, Kannel C, Paffenbarger RS Jr, Cupples LA. Heart rate and cardiovascular mortality: the Framingham Study. Am Heart J 1987;113:1489-94.

9. Cooney MT, Vartiainen E, Laatikainen T, Juolevi A, Dudina A, Graham IM. Elevated resting heart rate is an independent risk factor for cardiovascular disease in healthy men and women. Am Heart J 2010;159:612-9.

10. Mensink GB, Hoffmeister H. The relationship between resting heart rate and all-cause, cardiovascular and cancer mortality. Eur Heart J 1997;18:1404-10.

11. Diaz A, Bourassa MG, Guertin MC, Tardif JC. Long-term prognostic value of resting heart rate in patients with suspected or proven coronary artery disease. Eur Heart J 2005;26:967-74.

12. Jensen MT, Marott JL, Allin KH, Nordestgaard BG, Jensen GB. Resting heart rate is associated with cardiovascular and all-cause mortality after adjusting for inflammatory markers: the Copenhagen City Heart Study. Eur J Prev Cardiol 2012;19:102-8.

13. Custodis F, Baumhakel M, Schlimmer N, List F, Gensch C, Bohm M, et al. Heart rate reduction by ivabradine reduces oxidative stress, improves endothelial function, and prevents atherosclerosis in apolipoprotein E-deficient mice. Circulation 2008;117:2377-87.

14. Beere PA, Glagov S, Zarins CK. Retarding effect of lowered heart rate on coronary atherosclerosis. Science 1984;226:180-2.

15. Perski A, Olsson G, Landou C, de Faire U, Theorell T, Hamsten A. Minimum heart rate and coronary atherosclerosis: independent relations to global severity and rate of progression of angiographic lesions in men with myocardial infarction at a young age. Am Heart J 1992;123:609-16.

16. Heidland UE, Strauer BE. Left ventricular muscle mass and elevated heart rate are associated with coronary plaque disruption. Circulation 2001;104:1477-82.

17. Rogowski O, Shapira I, Shirom A, Melamed S, Toker S, Berliner S. Heart rate and microinflammation in men: a relevant atherothrombotic link. Heart 2007;93:940-4.

18. Inoue T, Iseki K, Iseki C, Kinjo K. Elevated resting heart rate is associat- ed with white blood cell count in middle-aged and elderly individuals without apparent cardiovascular disease. Angiology 2012;63:541-6.

19. O Hartaigh B, Bosch JA, Carroll D, Hemming K, Pilz S, Loerbroks A, et al. Evidence of a synergistic association between heart rate, inflammation, and cardiovascular mortality in patients undergoing coronary angiography. Eur Heart J 2013;34:932-41.

20. Whelton SP, Narla V, Blaha MJ, Nasir K, Blumenthal RS, Jenny NS, et al. Association between resting heart rate and inflammatory biomarkers (high-sensitivity C-reactive protein, interleukin-6, and fibrinogen) (from the Multi-Ethnic Study of Atherosclerosis). Am J Cardiol 2014;113:644-9.

21. Tracey KJ. The inflammatory reflex. Nature 2002;420:853-9.

22. Borovikova LV, Ivanova S, Zhang M, Yang H, Botchkina GI, Watkins LR, et al. Vagus nerve stimulation attenuates the systemic inflammatory response to endotoxin. Nature 2000;405:458-62.

23. Bernik TR, Friedman SG, Ochani M, DiRaimo R, Ulloa L, Yang H, et al. Pharmacological stimulation of the cholinergic antiinflammatory pathway. J Exp Med 2002;195:781-8.

24. Chiu JJ, Usami S, Chien S. Vascular endothelial responses to altered shear stress: pathologic implications for atherosclerosis. Ann Med 2009;41:19-28.

25. Himburg HA, Dowd SE, Friedman MH. Frequency-dependent response of the vascular endothelium to pulsatile shear stress. Am J Physiol Heart Circ Physiol 2007;293:H645-53.

26. De Keulenaer GW, Chappell DC, Ishizaka N, Nerem RM, Alexander RW, Griendling KK. Oscillatory and steady laminar shear stress differentially affect human endothelial redox state: role of a superoxideproducing NADH oxidase. Circ Res 1998;82:1094-101.

27. Walpola PL, Gotlieb AI, Cybulsky MI, Langille BL. Expression of ICAM1 and VCAM- 1 and monocyte adherence in arteries exposed to altered shear stress. Arterioscler Thromb Vasc Biol 1995;15:2-10.

28. Cheng JJ, Wung BS, Chao YJ, Wang DL. Cyclic strain enhances adhesion of monocytes to endothelial cells by increasing intercellular adhesion molecule-1 expression. Hypertension 1996;28:386-91.

29. Chappell DC, Varner SE, Nerem RM, Medford RM, Alexander RW. Oscillatory shear stress stimulates adhesion molecule expression in cultured human endothelium. Circ Res 1998;82:532-9. 